\title{
Deep Sulcus Sign
}

\author{
Ho Hyun Kim, Chan Yong Park, Hyun Min Cho \\ Department of Trauma Surgery, Pusan National University Hospital, Busan, Korea
}

The deep sulcus sign is a radiolucent lateral sulcus where the chest wall meets the diaphragm. In a supine film, it may be the only indication of a pneumothorax because air collects anteriorly and basally within the nondependent portions of the pleural space, as opposed to the apex when the patient is upright. The costophrenic angle is abnormally deepened when the pleural air collects laterally, producing the deep sulcus sign. We present a brief image of a trauma showing the deep sulcus sign indicating a pneumothorax.

Key Words: Pneumothorax; Diagnosis; X-Ray Film

\section{CASE}

A 56-year-old male pedestrian was brought to the emergency department after a high-speed road-traffic accident. He had transient hypotension and tachycardia, which improved after the administration of intravenous fluids. The physical examination revealed multiple orthopedic injuries in addition to trauma to the right chest, pelvis, and head. He underwent prompt intubation and sedation. Chest radiography with the patient in the supine position showed a deep sulcus sign (Fig. 1.), which was highly suggestive of a pneumothorax. Chest computed tomography confirmed a pneumothorax (Fig. 2.).

\section{DISCUSSION}

In the supine position, air in the pleural space distributes anteriorly and basally at nondependent portions and causes deepening of the lateral costophrenic angle,

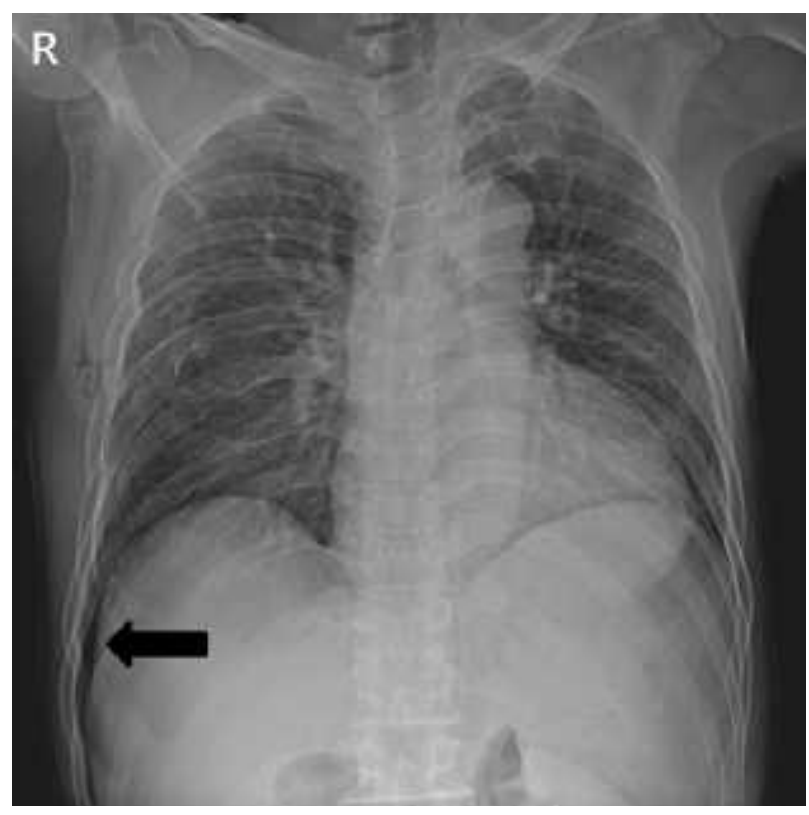

Fig. 1. Chest anteroposterior view radiograph shows a right pneumothorax with deep sulcus sign.

Received: May 2, 2016 Revised: July 3, 2016 Accepted: July 11, 2016

Correspondence to: Hyun Min Cho, Department of Trauma Surgery, Pusan National University Hospital, 179, Gudeok-ro, Seo-gu, Busan, Korea

Tel: 82-51-240-7369, Fax: 82-51-240-7719, E-mail: csking1@daum.net

Copyright (c) 2016 Korean Association for Research, Procedures and Education on Trauma. All rights reserved.

@This is an open-access article distributed under the terms of the Creative Commons Attribution Non-Commercial License (http://creativecommons.org/ licenses/by-nc/4.0) which permits unrestricted noncommercial use, distribution, and reproduction in any medium, provided the original work is properly cited 

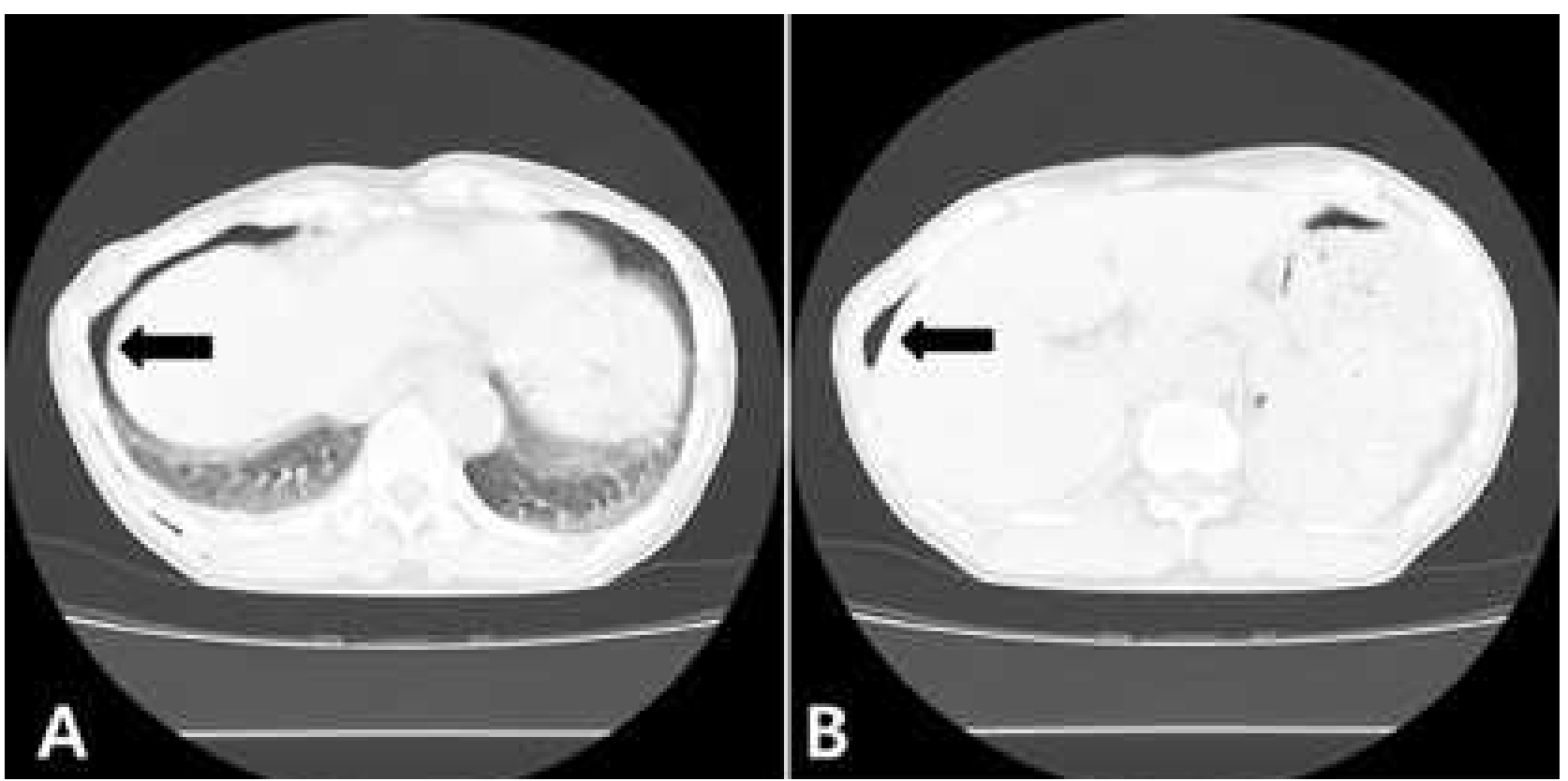

Fig. 2. Chest computed tomography revealed a pneumothorax.

producing the deep sulcus sign (1-3). The deep sulcus sign is an important clue for diagnosing pneumothorax.

\section{CONFLICT OF INTEREST}

No potential conflict of interest relevant to this article was reported.

\section{REFERENCES}

1. Jeng MJ, Lee YS, Tsao PC, Soong WJ. Neonatal air leak syndrome and the role of high-frequency ventilation in its prevention. Journal of the Chinese Medical Association : JCMA. 2012;75(11):551-9.

2. Sabbar S, Nilles EJ. Images in clinical medicine. Deep sulcus sign. The New England journal of medicine. 2012; 366(6):552.

3. Wang SH, Wei KY, Liu YC, Huang CH. The deep sulcus sign indicates free air in the abdomen. Internal and emergency medicine. 2015;10(8):1033-4. 